\title{
La littérature jeunesse en petite enfance : l'équipe comme contexte de formation
}

Children's literature in early childhood: the team as a training context

\section{Catherine Peresse Schroeder et Rawad Chaker}

\section{OpenEdition}

Journals

\section{Édition électronique}

URL : https://journals.openedition.org/trema/6686

DOI : $10.4000 /$ trema.6686

ISSN : 2107-0997

\section{Éditeur}

Faculté d'Éducation de l'université de Montpellier

Référence électronique

Catherine Peresse Schroeder et Rawad Chaker, « La littérature jeunesse en petite enfance : l'équipe comme contexte de formation », Tréma [En ligne], 55 | 2021, mis en ligne le 20 octobre 2021, consulté le 13 décembre 2021. URL : http://journals.openedition.org/trema/6686 ; DOI : https://doi.org/ 10.4000/trema.6686

Ce document a été généré automatiquement le 13 décembre 2021.

Trema 


\section{La littérature jeunesse en petite enfance : l'équipe comme contexte de formation}

Children's literature in early childhood: the team as a training context

Catherine Peresse Schroeder et Rawad Chaker

\section{Introduction}

1 De nombreuses études montrent que la lecture de livres dans les lieux d'accueil de la petite enfance entraine des résultats positifs sur l'acquisition de vocabulaire et sur le développement du langage des enfants (Arnold et al., 1994; Reese et Cox, 1999 ; Wasik et Bond, 2001 ; Whitehurst et al., 1994 ; Whitehurst et Lonigan, 1998 ; cités par Flowers, Girolametto, Weitzman, et Greenberg, 2007). Pourtant, la formation en France des professionnelles ${ }^{1}$ petite enfance est courte et le livre n'est que peu présent dans ces cursus (ASP, 2020). En effet, dans les référentiels des diplômes du Certificat d'Aptitude Professionnelle « Accompagnant éducatif petite enfance » et du diplôme professionnel d'auxiliaire de puériculture, le livre n'est pas mentionné. Ces référentiels indiquent que les professionnelles doivent être capable de proposer des activités d'éveil aux enfants mais les centres de formation ont le choix d'orienter ce module comme ils le souhaitent, l'initiation à la littérature jeunesse n'étant pas proposée par tous. Les professionnelles manquent souvent de temps, de formations et d'outils pour proposer le livre de manière adéquate à l'enfant (Aragon, 2013, pp. 70-77; Meuret-Campfort, 2014, pp. 81-108 ; Rayna, 2003, pp. 30-41). Il existe de nombreux ouvrages et articles sur l'intérêt de lire des livres aux enfants (Bergeron, 2009), notamment sur leur rôle dans la lutte contre les inégalités socioculturelles (Bonnafé, 2001) et sur l'importance des structures d'accueil pour le développement des enfants des milieux défavorisés a été soulignée (Bigras et Lemay, 2012). Mais peu de recherches francophones se sont penchées sur la formation des professionnelles petite enfance à l'utilisation du livre jeunesse (constat de recherches sur Cairn.info ou Google Scholar). Ainsi, le but de cette 
recherche inductive et exploratoire est d'enquêter sur le discours des professionnelles petite enfance sur l'usage et la formation au livre jeunesse, en prenant en compte les contraintes du terrain et du métier. Quels sont les éléments à prendre en compte ? Par quel biais une formation professionnelle pourrait-elle se mettre en place? L'intérêt d'un tel sujet réside par ailleurs dans les enjeux sociaux liés à la littérature jeunesse et à la petite enfance. En effet, pour Bonnafé (2019), la littérature jeunesse est un outil essentiel dans cette lutte contre les inégalités sociales. Elle permet le développement d'une culture commune (Nières-Chevrel, 2002, pp. 97-114) et de découvrir de nouveaux mots, notamment chez un public allophone (Piselli et Brengou, 2019, pp. 123-133). L'étude Abecedarian effectuée en 1972 aux États-Unis (citée par Noblecourt, 2014) a par exemple montré l'impact d'un programme d'éveil en crèche sur une population d'enfants pauvres. Cette étude, basée sur l'apport du livre, le langage et le soutien à la parentalité, montre que les enfants ayant bénéficié de ce programme accèdent aux études supérieures et à des emplois qualifiés par rapport à ceux n'en ayant pas bénéficié. Toutes ces raisons nous mènent à affirmer qu'il est essentiel de se pencher sur les conditions d'une formation professionnelle à l'usage du livre pour enfant.

2 En effet, proposer le livre aux enfants par les professionnelles petite enfance ne va pas forcément de soi: "lire des livres aux bébés est loin d'être une évidence.» (Chavepeyer, 2020, p. 159). De plus, « les puéricultrices fréquentent rarement les lieux culturels : ouvrir la porte d'une bibliothèque se fait parfois » (ibid.) mais n'est pas chose commune. En effet, les professionnelles voient souvent le livre comme étant une «activité " à "animer » (ibid.). Or, l'art, dont fait partie la littérature jeunesse, n'implique pas une démarche de "faire faire " mais une démarche d'«être » (ibid.) partant de l'expérimentation, de l'observation, du ressenti, de l'échange. Proposer le livre en petite enfance nécessite d'avoir certaines connaissances et compétences. En effet, l'utilité du livre pour le jeune enfant n'est plus à démontrer mais la manière de l'amener à l'enfant est particulière à cette tranche d'âge: libre exploration, libre disposition, libre choix, lecture individuelle, utilisation des sens, attention conjointe (Bonnafé 2019 ; Cabrejo-Parra, 2019 ; CREDE, 2021 ; Thollon-Behar et Ignacchiti, 2019).

Former les professionnelles est donc indispensable mais nécessite, comme dans tout cadre de travail, de prendre en compte la réalité professionnelle. En effet, les professionnelles petite enfance peuvent avoir tendance à se sentir appartenir à un établissement et donc à une équipe plus qu'à une profession (Marchand Montanaro, 2013). Nous pouvons donc distinguer l'équipe professionnelle petite enfance comme un groupe social. D'après De Coster, le groupe social est une "formation sociale à l'intérieur de laquelle les individus sont en interaction selon des règles fixées [...] partagent le sentiment de constituer une entité particulière en sorte que les membres pourraient s'y reconnaitre comme tels » (1990, p. 125). La formation continue passerait donc par la socialisation professionnelle en équipe et elle concourrait à favoriser un sentiment d'appartenance sociale qui est étroitement lié à la motivation et à la participation au travail (Deci et al., 1989, 1993 cités par Richer et Vallerand, 1998). Le cadre théorique choisi, celui de l'Approche de l'Action Raisonnée (AAR : Fishbein et Ajzen, 2010) peut permettre de comprendre les motivations qui poussent les adultes à suivre des formations continues (cf. les études de Hopstock, 2008 ; Diethert, Weisweiler, Frey et Kerschreiter, 2015), tout comme les attitudes ou les normes subjectives (Norwich et Duncan, 1990), et les croyances (Becker et Gibson, 1998) qui sous-tendent le comportement des sujets. 
4 Notre question principale de recherche est: comment orienter la formation à l'usage du livre pour permettre un investissement efficace des professionnelles petite enfance ? Nous émettons l'hypothèse que les professionnelles petite enfance expriment la nécessité d'adopter une approche centrée sur l'équipe pour être formées efficacement à l'utilisation de la littérature jeunesse.

5 Nous étudierons le concept de formation continue par l'analyse de pratiques, la socialisation en formation et l'Approche de l'Action Raisonnée. Cette approche nous servira de cadre théorique et méthodologique pour notre d'enquête auprès des professionnelles petite enfance. Nous étudierons sur le terrain, de manière inductive et exploratoire, les processus de professionnalisation, leurs attitudes vis-à-vis de la formation à l'usage du livre en petite enfance et les outils pouvant favoriser la formation. Nous proposerons enfin des axes de formation à l'usage du livre en petite enfance.

\section{La formation continue}

\section{La formation professionnelle à la littérature jeunesse}

6 La formation professionnelle à la littérature jeunesse nécessite de prendre en compte le terrain au sein duquel évolue les professionnelles. En effet, des injonctions théoriques et normatives n'auraient pas de sens pour ces professionnelles qui, souvent, se sentent "dénigrées dans leurs pratiques, niées dans leurs savoirs issus de l'expérience, attaquées dans leurs valeurs » (Patras Mériaux et Desrumaux, 2018, p. 52), si la réalité du terrain et les spécificités du contexte ne sont pas prises en compte. Il semble dès lors indispensable de proposer une démarche collective et collaborative (Vinatier, Filliettaz et Laforest, 2018) pour former les professionnelles in situ, en fonction des problématiques locales. L'essor récent de l'intérêt scientifique pour la formation professionnelle des adultes, et plus particulièrement celle concernant les métiers éducatifs (Lussi Borer, Durand et Yvon, 2015), repose sur l'analyse de pratiques permettant le développement professionnel (Marcel, 2002 ; Filliettaz et Billett, 2015). Ce concept d'analyse de pratiques repose sur la notion de travail réel en situation concrète de terrain. Transposé à la littérature jeunesse, ce principe engendre la nécessité d'une formation professionnelle partant du vécu de l'expérience afin de rendre les professionnelles actrices: les interactions sociales collectives (Mondada, 2006) sont indispensables pour permettre le développement des compétences professionnelles et des savoirs professionnels construits collectivement de manière située.

\section{L'identité professionnelle par la formation}

7 L'identité sociale d'un individu ne se résume pas à son appartenance à un groupe social mais à plusieurs. La socialisation s'effectue entre « des processus sociaux (pluriels et divers) et des parcours individuels (multiples et plus ou moins complexes) (Dubar, 2015, p. 15-16). Parmi cette multiplicité de construction de l'identité sociale, «la dimension professionnelle a acquis une importance particulière ». En effet, « l'emploi ... le travail ... la formation» entrainent des «transformations identitaires » et " conditionnent la construction des identités sociales» (ibid.). Ainsi, le lieu de travail, l'interaction entre les membres de l'équipe professionnelle et les formations en son 
sein développent une construction identitaire professionnelle amenant à une culture commune. Moore (1969) « établit une distinction importante entre les normes idéales et formelles transmises par la formation et les normes pratiques et informelles consolidées par l'expérience du travail » (Moore cité par Dubar, 2015, p. 143-160). Ainsi, la socialisation professionnelle peut concourir à une formation identitaire professionnelle commune. C'est pourquoi la formation pratique de l'équipe au sein de leur action peut permettre la construction d'une identité partagée, permettant ainsi de mêler la théorie et la pratique comme définies par Moore, tout en prenant en compte les particularismes en termes de culture et d'éthique professionnelles liées à un contexte local.

\section{Entre résistance et intérêt}

8 D'après l'Approche de l'Action Raisonnée (AAR) (Fishbein et Ajzen, 2010), le comportement d'une personne est notamment déterminé par son intention d'adopter ou non un comportement, qui est elle-même caractérisée par les normes subjectives (Norwich et Ducan, 1990). L'intérêt de l'AAR est de pouvoir étudier, dans le cadre d'une étude qualitative exploratoire basée sur un échantillon réduit, les comportements et représentations individuels tout en les inscrivant dans un champ plus large par l'étude des normes perçues (facteurs liés à l'équipe et au collectif) et des facteurs externes. Les normes subjectives sont déterminées par ce que les autres pensent de ce que l'on devrait faire et par la motivation à se soumettre (norme injonctive). Certaines conduites sont donc influencées par une forte identité socio-culturelle (Bagozzi et Lee, 2002), liée à une culture professionnelle spécifique et située. A vu de ces éléments, nous réfléchissons ainsi à la manière de faciliter la formation des professionnelles, professionnaliser et stabiliser localement leur usage du livre et favoriser leurs prédispositions vers une telle formation.

\section{Méthodologie}

Notre cadre théorique met en avant la conduite individuelle mais également le rôle de l'entourage dans la prise de décision. Ainsi, après avoir étudié les savoirs construits des professionnelles, nous cherchons, par une première étude de cas exploratoire auprès de professionnelles petite enfance, à savoir dans quelle mesure la socialisation au sein de l'équipe et la formation expérientielle sont à prendre en compte pour former efficacement les professionnelles petite enfance à l'usage du livre jeunesse? Les professionnelles petite enfance se sentant appartenir à un groupe, nous émettons les hypothèses que la formation en équipe peut offrir un cadre efficace pour les former et qu'une interaction forte au sein de l'équipe favorisera la mise en place d'une réflexivité bénéfique pour une utilisation adaptative du livre jeunesse. Il s'agit donc ici d'évaluer le ressenti des professionnelles quant à ce mode de formation en équipe, de manière inductive et exploratoire.

\section{Échantillon et procédure}

10 Notre premier entretien s'est déroulé dans les locaux d'une association proposant des formations sur le livre aux professionnelles petite enfance en région parisienne depuis 
plusieurs années. Cet entretien avec la formatrice, croisé avec la littérature sur le sujet, nous procure un premier point de vue prescriptif (de par son rôle) sur les savoirs et savoir-faire adaptés en matière de littérature jeunesse et les pratiques de formation. Cela nous permet également d'obtenir quelques éléments de compréhension des principaux obstacles rencontrés par les professionnelles et de connaitre leurs retours, à travers le témoignage de la formatrice, sur les méthodes de formation appréciées.

11 Nous avons effectué deux autres entretiens au sein d'un multi-accueil situé en zone sensible dans le quartier du Petit Nanterre en région Parisienne, où toutes les origines sociales et modèles familiaux sont représentés (favorisés, allophones, défavorisés, difficultés financières, éducatives...). Nous avons choisi d'interroger deux professionnelles de terrain exerçant en multi-accueil depuis plusieurs années. Ainsi, par leur expérience conséquente, elles ont vécu plusieurs situations d'utilisation du livre et ont accueilli des enfants d'origines socioculturelles variées. Nous avons choisi d'interroger une professionnelle n'ayant pas bénéficié de formation supplémentaire autour du livre afin d'obtenir un point de vue non influencé par des apports de connaissances et une professionnelle ayant suivi la formation autour du livre afin de les mettre en confrontation et d'évaluer les divergences entre les réponses des deux professionnelles ce qui nous permet de savoir si la formation a un impact ou non. Ces entretiens vont nous permettre de cerner les attentes et les connaissances des professionnelles petite enfance concernant la littérature jeunesse, d'accéder à la façon dont elles se représentent le livre et l'interaction enfant-livre, et, enfin leur manière d'envisager la formation professionnelle sur ce sujet. Cette première approche, bien que réduite, nous permettra d'effectuer une étude exploratoire sur un sujet peu étudié - la littérature jeunesse - avec une approche située et contextualisée. Nous avons construit notre méthodologie à partir de grilles d'entretiens semi-directifs.

\section{Analyse thématique et catégorielle}

12 Nous avons opéré une catégorisation des discours selon la définition de Bardin (2013): "classification d'éléments constitutifs d'un ensemble par différenciation puis regroupement par genre (analogie) d'après des critères définis afin de fournir, par condensation, une représentation simplifiée des données brutes» (Bardin, 2013). Pour procéder à ce classement thématique, nous avons défini des variables et des indicateurs, relié les éléments des entretiens à ces indicateurs et recensé les occurrences des éléments afin de dégager les thèmes principaux (voir tableaux 1 à 3 ).

\section{Résultats}

\section{Thème 1 : " état des savoirs et des pratiques professionnels sur la littérature jeunesse, son rôle dans les inégalités, l'enfant allophone et/ ou défavorisé ».}

13 Les connaissances et comportements ont été déterminés comme adaptés par rapport aux éléments énoncés par la formatrice se retrouvant dans le programme de formation à la littérature : lecture individuelle (Bonnafé, 2019, pp. 41-54 ; Cabrejo-Parra, 2019), utilisation d'albums à structure itérative, inclusion des parents, livres en libre-accès pour favoriser la libre manipulation (Bonnafé, 2019, p. 47 ; Thollon-Behar et Ignacchiti, 
2019, p. 40), choix des enfants (CREDE, 2021), prise en compte de l'enfant dans l'adaptabilité de la voix, de la gestuelle, observation des enfants (Enfance et Musique, 2020), découverte par l'enfant, nécessité d'un milieu riche (Best, 1973). L'ensemble de ces éléments se retrouvent dans le programme de formation de l'association. Les résultats du tableau 1 nous montrent que les connaissances semblent partiellement acquises (10 et 12 occurrences selon les professionnelles) et que les comportements de la professionnelle 2 ayant eu la formation autour du livre sont plus adaptés (20 occurrences de comportement adapté et 8 occurrences de comportements inadaptés) par rapport à la professionnelle 1 (7 et 9 occurrences). Seule la libre-accessibilité et l'observation sont évoquées par les professionnelles. De nombreux marqueurs d'hésitation sont présents, surtout chez la professionnelle 1 sans formation (36 occurrences vs 16 occurrences chez la professionnelle 2 avec formation).

Tableau 1 : concordance des indicateurs et des éléments d'entretien sur le thème 1

\begin{tabular}{|c|c|c|c|}
\hline \multirow[t]{2}{*}{ Indicateurs } & \multicolumn{3}{|c|}{ Exemples d'unités de langage par personne interviewée } \\
\hline & Formatrice & Professionnelle 1 & Professionnelle 2 \\
\hline $\begin{array}{l}\text { Connaissances } \\
\text { acquises }\end{array}$ & $\begin{array}{l}\text { Les professionnelles } \\
\text { «savent beaucoup de } \\
\text { choses » } \\
\text { «1'ont déjà vécu» }\end{array}$ & $\begin{array}{l}\text { Utilité du livre : « langage », } \\
\text { " reconnaitre », « apprendre } \\
\text { de nouveaux mots », } \\
\text { " familiariser » } \\
\text { Attitude de l'enfant : «il } \\
\text { entend, il est à côté », }\end{array}$ & $\begin{array}{l}\text { Utilité du livre : « une } \\
\text { interaction », « les fait } \\
\text { parler », « participer », « très } \\
\text { utile », « langage » }\end{array}$ \\
\hline $\begin{array}{l}\text { Connaissances non } \\
\text { acquises }\end{array}$ & $\begin{array}{l}\text { Les professionnelles } \\
\text { ont des } \\
\text { connaissances } \\
\text { «cloisonnées » }\end{array}$ & $\begin{array}{l}\text { Le livre ne peut pas lutter } \\
\text { contre les inégalités «A cet } \\
\text { âge-là je ne pense pas trop », } \\
\text { ne sait pas pourquoi elle finit } \\
\text { un livre « il n’y a pas de } \\
\text { fin » }\end{array}$ & \\
\hline $\begin{array}{l}\text { Compétences et } \\
\text { comportements } \\
\text { adéquats liés à la } \\
\text { littérature et aux } \\
\text { recommandations } \\
\text { de la formatrice }\end{array}$ & & $\begin{array}{l}\text { Utilisation du paraverbal : } \\
\text { " gestes », « signe de } \\
\text { manger » (après relance), à } \\
\text { la « demande }\end{array}$ & $\begin{array}{l}\text { «à disposition », } \\
\text { « demandeurs », «c'est eux } \\
\text { qui demandent», "je } \\
\text { m'installe, ça les attire », } \\
\text { observe les signes montrant } \\
\text { que l'enfant écoute « ils me } \\
\text { parlent de loin » }\end{array}$ \\
\hline $\begin{array}{l}\text { Divergences entre } \\
\text { les } \\
\text { recommandations } \\
\text { de la formatrice et } \\
\text { l'utilisation du livre }\end{array}$ & $\begin{array}{l}\text { N'utilisent pas de CD } \\
\text { dans la langue } \\
\text { maternelle, pas } \\
\text { d'utilisation de livres } \\
\text { à structure itérative, } \\
\text { pas d'inclusion des } \\
\text { parents }\end{array}$ & $\begin{array}{l}\text { « regrouper », « en groupe », } \\
\text { «je ne fais que répéter, } \\
\text { répéter », }\end{array}$ & $\begin{array}{l}\text { «je leurs dis venez », « pour } \\
\text { se calmer », « temps calme », }\end{array}$ \\
\hline $\begin{array}{l}\text { Marqueurs de } \\
\text { connaissances } \\
\text { morcelés }\end{array}$ & & $\begin{array}{l}\text { «e ne sais pas » } \mathrm{x} 5 \text { (qu'est- } \\
\text { ce qu'un album jeunesse, } \\
\text { comptines en langue } \\
\text { maternelle, compétences de } \\
\text { l'enfant allophone, ne sait } \\
\text { pas si elle fait des erreurs, } \\
\text { inclusion des parents) } \\
\text { Hésitations et « euh... »: } \\
\text { x27 } \\
\text { Nombreuses relances x9 }\end{array}$ & $\begin{array}{l}\text { «Je ne sais pas » X2 (qu'est-ce } \\
\text { qu'un album jeunesse, } \\
\text { compétences de l'enfant } \\
\text { allophone) } \\
\text { Euh... x16 }\end{array}$ \\
\hline
\end{tabular}

\section{Thème 2 : motifs d'engagement}

Le tableau 2 nous permet de comprendre comment les professionnelles se positionnent par rapport à la formation (résistance/acceptabilité). En effet, « les facteurs conatifs (attitudes, motivation, volition) » sont des paramètres cruciaux à prendre en compte pour appréhender le "choix et l'orientation des conduites » en vue d'un engagement dans une formation (Carré, 2015). Sur la motivation (tableau 2), les résultats nous montrent que les professionnelles sont motivées à se former (10 et 9 occurrences contre 1 et 0 occurrence d'amotivation). Elles ont un intérêt certain pour la lecture et l'apport du livre (attitude instrumentale et expérientielle). Néanmoins, nous notons que la formatrice rapporte des sentiments négatifs par rapport au livre de la part des professionnelles qu'elle a formé (4 occurrences). De plus, la distance physique entre le 
lieu d'exercice et le lieu de formation a été rapportée comme étant un obstacle pour certaines professionnelles. La formatrice a cependant rencontré peu de réfractaires ce qui nous indique une acceptabilité de la formation de la part des professionnelles. Les normes perçues (injonctives et descriptives) nous indiquent une acceptabilité de la formation, comme expliqué dans l'AAR (Fishbein et Ajzen, 2010), par la volonté d'imiter les collègues (professionnelle 1).

Tableau 2 : concordance des indicateurs et des éléments d'entretien sur le thème 2

\begin{tabular}{|c|c|c|c|}
\hline \multirow[t]{2}{*}{ Indicateurs } & \multicolumn{3}{|c|}{ Exemples d'unités de langage par personne interviewée } \\
\hline & Formatrice & Professionnelle 1 & Professionnelle 2 \\
\hline $\begin{array}{l}\text { Degré d'intérêt } \\
\text { pour le livre } \\
\text { pour soi et la } \\
\text { formation }\end{array}$ & $\begin{array}{l}\text { Elles « ressentent } \\
\text { l'utilité »; } \\
\text { « sont convaincues } \\
\text { que c'est } \\
\text { important »; se } \\
\text { demandent } \\
\text { « comment faire » } \\
\text { « de plus en plus } \\
\text { [...] ne sont pas } \\
\text { convaincus» }\end{array}$ & $\begin{array}{l}\text { Intérêt du livre « pour la mémoire » } \\
\mathrm{x} 2 \\
\text { Intérêt de la formation : « oui », } \\
\text { «pour avoir plus de connaissances », } \\
\text { Pas d'indicateurs de désintérêt: }\end{array}$ & $\begin{array}{l}\text { Intérêt de la formation : } \\
\text { «c'est intéressant } », \text { " on } \\
\text { apprend } », ~ « \text { pratique } », \\
\text { « façon de lire » }\end{array}$ \\
\hline $\begin{array}{l}\text { Possibilité de } \\
\text { se former }\end{array}$ & & $\begin{array}{l}\text { Ne souhaite pas se déplacer, ne } \\
\text { connait pas de formations sur le livre }\end{array}$ & $\begin{array}{l}\text { N'a pas eu de difficultés à } \\
\text { se former, n'est pas dérangé } \\
\text { par la distance }\end{array}$ \\
\hline $\begin{array}{l}\text { Sentiment par } \\
\text { rapport au livre }\end{array}$ & $\begin{array}{l}\text { «livres = école, } \\
\text { livres }= \\
\text { apprentissage } » \\
\text { « violence } » x 3\end{array}$ & «ça fait du bien » & «J'aime beaucoup $» \mathrm{x} 2$ \\
\hline $\begin{array}{l}\text { Normes } \\
\text { perçues }\end{array}$ & & volonté d'imiter les collègues & \\
\hline $\begin{array}{l}\text { Acceptabilité } \\
\text { de la formation }\end{array}$ & $\begin{array}{l}\text { A rencontré peu de } \\
\text { réfractaires }\end{array}$ & & \\
\hline
\end{tabular}

\section{Thème 3 : Quels procédés pour la formation?}

Les résultats sur les méthodes (tableau 3) nous montrent que l'équipe (19 occurrences) fait partie des moyens principaux sur lesquels se baser. La formation sur le terrain (15 occurrences) et le projet (16 occurrences) sont également des leviers de formation qui permettent de rendre l'action durable et efficace. La formation semble devoir s'orienter vers l'observation (48 occurrences), la posture (31 occurrences), la perception (17 occurrences), la parole et l'écoute (11 occurrences) et la composante artistique (8 occurrences). La majorité de ces orientations peuvent s'effectuer sur le terrain, par des analyses expérientielles. L'inclusion de la famille (citée 22 fois par la formatrice et 0 fois par les professionnelles) semble également être un point à travailler. 
Tableau 3 : concordance des indicateurs et des éléments d'entretien sur le thème 3

\begin{tabular}{|c|c|c|c|}
\hline \multirow[t]{2}{*}{ Indicateurs } & \multicolumn{3}{|c|}{ Exemples d'unités de langage par personne interviewée } \\
\hline & Formatrice & Professionnelle 1 & Professionnelle 2 \\
\hline $\begin{array}{l}\text { Marqueurs de } \\
\text { positivité }\end{array}$ & Travail d'《 équipe » x 9, « échanges » & $\begin{array}{l}\text { « projet } » \times 2 \\
\text { « chacune apporte } »\end{array}$ & $\begin{array}{l}\text { avoir « un autre } \\
\text { regard» } \\
\text { les « collègues » }\end{array}$ \\
\hline $\begin{array}{l}\text { Préconisations } \\
\text { de la formatrice }\end{array}$ & 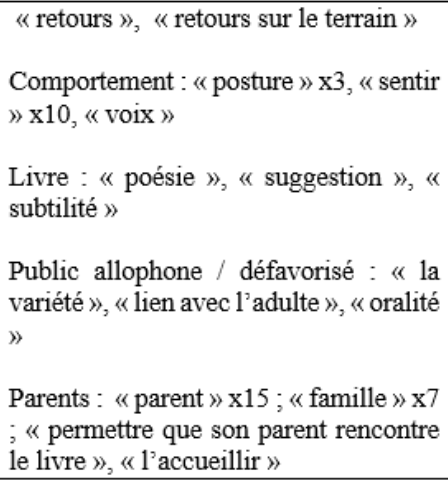 & & \\
\hline $\begin{array}{l}\text { Moyens de } \\
\text { formation }\end{array}$ & $\begin{array}{l}L^{2} \text { « observation } \ldots \text { observé } » \times \text { X } \\
\text { « voir ... vu » x } 24 \text {, « yeux ... œil » }\end{array}$ & & \\
\hline $\begin{array}{l}\text { Temps } \\
\text { d'utilisation }\end{array}$ & & $\begin{array}{l}\text { Quotidien } \\
\text { livres « à disposition» }\end{array}$ & $\begin{array}{l}\text { « projet» } \\
\text { hebdomadaire } \\
\text { livres « à disposition », }\end{array}$ \\
\hline Projet & & Mis en place et régulier & $\begin{array}{l}\text { partenariat avec la } \\
\text { «bibliothèque » }\end{array}$ \\
\hline
\end{tabular}

\section{Discussion}

\section{Des savoirs et pratiques partiels}

Les savoirs et pratiques à propos du livre, des inégalités, de l'enfant et du public allophone ou défavorisé sont partiellement intégrés au regard de la théorie sur le sujet (Bonnafé, 2019; Best, 1973; Thollon-Behar et Ignacchiti, 2019) et du discours de la formatrice. Les professionnelles possèdent certaines connaissances sur l'utilisation du livre : mise à disposition des livres, finir une histoire débutée, respect du rythme de l'enfant (indispensable selon Dethier, Bosse-Platière, Fleury, et Loutre Du Pasquier, 2011 ; pp. 139-197) ; mais ne savent souvent pas pourquoi elles agissent ainsi. Pourtant, la pratique réflexive est une dimension essentielle de l'agir professionnel (Schön, 1994).

17 Les comportements des professionnelles n'apparaissent pas toujours comme adaptés aux prescriptions données par la formatrice et dans la littérature (ne pas imposer le livre, utilisation en temps individuel, ne pas utiliser le livre pour calmer un groupe, s'adapter à l'enfant) : par exemple, elles utilisent encore le livre pour canaliser les enfants. Les professionnelles ne savent souvent pas la raison de leurs agissements ce qui ne leur permettent pas d'adapter leurs propositions en fonction des enfants. Elles ne semblent pas réfléchir à leurs pratiques autour du livre. Par exemple, une professionnelle dit énoncer le nom de l'auteur aux enfants car elle l'a appris en formation mais elle ne sait pas pourquoi elle agit ainsi. Leurs connaissances déclaratives sur l'enfant allophone ou défavorisé sont partielles. Nous remarquons que la professionnelle ayant eu la formation a des connaissances et un comportement plus adaptés; elle comprend que la lecture permet aux enfants d'obtenir les codes sociolinguistiques et de faire évoluer leur habitus. Elle semble agir face à ce public de 
manière adéquate : utilisation du rythme, de la mélodie, création du lien entre l'adulte et l'enfant ; mais elle n'utilise pas tous les outils possibles : livres à structure itérative, livres ou comptines dans la langue des enfants permettant le transfert vers une nouvelle langue (Auger, 2011), utilisation des compétences d'oralité et des capacités linguistiques et intellectuelles des enfants allophones (Cummins, 2001 cité par Auger, 2011). Elle ne semble pas adapter son approche en fonction de l'enfant. La professionnelle sans formation n'a pas de connaissances et de compétences spécifiques face à ce public (elle se sert d'imagiers, use de la répétition).

Plus que des savoirs, nous voyons ici que c'est une réelle réflexivité qui manque aux professionnelles, réflexivité pouvant être construite en équipe. En effet, ces connaissances et comportements sont essentiels pour amorcer une utilisation du livre adéquate, mais l'appropriation de ces savoirs et savoir-faire de manière individuelle ne suffit pas pour permettre une offre conjointe et adaptée de la littérature jeunesse en structure d'accueil.

\section{L'équipe comme source d'engagement}

19 Les professionnelles sont motivées pour se former et trouvent un intérêt au livre pour elles-mêmes et pour les enfants. Elles cherchent à acquérir des compétences sur son utilisation. La formatrice nous indique que les professionnelles souhaitent savoir comment faire pour intéresser l'enfant. Les seules réfractaires à l'utilisation du livre le sont du fait du rapport qu'elles ont elles-mêmes eu avec le livre et qui leur renvoie un sentiment d'échec et une violence liée à leurs propres apprentissages.

La professionnelle 2 trouve une source de motivation parmi ses collègues : elle utilise le livre si ses collègues l'utilisent, confirmant ainsi notre introduction théorique. Le sentiment d'appartenance au groupe est important pour la professionnelle. L'interaction avec ses collègues et les échanges sur les manières de procéder peuvent donc être instructifs pour elle. Ainsi, une vision commune partagée (Akram et al., 2016 cité par Claridge, 2018) semble être nécessaire pour améliorer les pratiques. La formatrice nous indique que de nombreuses professionnelles se sentent jugées lorsqu'elles utilisent le livre; elles ont l'impression de ne pas être utiles à l'enfant. Les normes perçues semblent importantes pour de nombreuses professionnelles. Les collègues de travail sont donc une composante essentielle à prendre en compte dans le cadre de la formation autour du livre jeunesse. L'équipe semble donc être un levier pour permettre d'amorcer une réflexion de groupe.

\section{L'équipe comme cadre de formation}

21 En effet, les professionnelles interrogées mettent l'accent sur l'importance d'une formation en équipe: l'interaction avec l'environnement social semble importante pour permettre une formation efficace. Les connaissances et comportements ne sont pas toujours adaptés: le public qui n'a pas de livre chez lui n'écoute pas, les enfants doivent rester assis, contrairement aux préconisations indiquant qu'un enfant bouge durant une lecture et qu'il est nécessaire de le laisser faire (Piselli et Brengou, 2019, pp. 123-133), le livre sert à canaliser ce qui peut gêner l'émergence de l'utilisation du livre comme geste professionnel. La formation en équipe permettrait alors de se décentrer de son propre mode de pensée et d'effectuer un travail de différenciation 
entre ses représentations et la réalité objective (Bosse-Platière, Dethier, Fleury, et Loutre Du Pasquier, 2011, pp. 253-254). La formation en équipe permettrait à chacune de connaitre le point de vue de l'autre, tout en étant guidée par une formatrice spécialisée sur le sujet, afin de partager un même mode de pensée autour du livre, les mêmes savoirs et les mêmes compétences. Disposer des mêmes informations faciliterait l'utilisation du livre car l'ensemble de l'équipe se situera sur la même ligne de conduite et acceptera les actions de chacune de ses membres par compréhension des gestes professionnels.

Il apparait ainsi comme nécessaire de former les professionnelles au livre petite enfance via une " écoute attentive des tâtonnements de l'enfant» et une «interaction adaptée » pour «lui offrir les fonctionnements sémantico-syntaxiques adéquats à son désir d'expression et à la situation » (Vertalier, 2006, p. 57). L'observation en équipe peut également favoriser la compréhension de ce qui se joue chez l'enfant dans une relation de lecture par l'échange sur les expériences vécues. Nous observons, d'après la formatrice, que la formation en équipe permet un travail sur le terrain. Les professionnelles évoquent le partage et l'expérience de chacune. Ces éléments, associés aux connaissances théoriques et aux préconisations de la formatrice et de la littérature, permettent ainsi une réflexivité des professionnelles et une adaptabilité de leurs pratiques en fonction du contexte et de l'enfant.

D'après les différents discours recueillis, nous pouvons noter l'importance de l'équipe en tant que support de la formation et composante permettant de faire un atout des normes perçues localement telles que définies dans l'AAR (Fishbein et Ajzen, 2010). L'équipe, réfléchissant elle-même sur ses pratiques, serait ainsi actrice de sa formation et de ses apprentissages, dépassant la simple injonction prescriptive. Une telle dynamique permettrait de prendre en compte l'expérience professionnelle, sociale ou personnelle des membres de l'équipe (Depover et Marchand, 2002). La nécessaire professionnalisation à l'usage du livre petite enfance semble donc devoir passer par la socialisation de l'équipe et le partage d'une identité commune afin d'objectiver et de stabiliser ses usages dans le contexte professionnel de l'accueil du jeune enfant.

\section{Conclusion : implications pratiques}

\section{Suggestions d'axes de formation à l'usage réflexif du livre jeunesse}

D'après nos résultats, les professionnelles et la formatrice orientent les besoins vers une formation partagée par l'équipe. En effet, celle-ci permettrait un partage, un autre regard et un projet conjoint qui favoriserait l'utilisation quotidienne du livre ainsi que les interactions entre les professionnelles. À leur tour, ces interactions permettraient d'engendrer plusieurs situations utiles à l'utilisation du livre, dépassant le cadre des seules préconisations théoriques :

- L'apparition d'une personne ressource, parmi les membres de l'équipe, permettant de poser un regard critique et bienveillant sur les pratiques lors de temps d'observation.

- Des analyses de pratique en groupe, permettant d'amorcer un «travail de transformationévolution du regard et des pratiques » (Bosse-Platière et al., 2011, p. 252), en veillant à distribuer parole et rôles selon la composition de l'équipe.

-L'accompagnement à la réflexivité (Bouissou et Brau-Anthony, 2005 ; Lafortune, 2015), qui apparait justement comme un levier fondamental, au niveau individuel comme au niveau 
collectif, pour la construction identitaire (Faingold, 2006) et pour identifier via des habiletés métacognitives l'ensemble des savoirs tacites ou cachés qui structurent la réflexion du sujet (Schön, 1994).

-L'émergence de personnes supports qui pourraient aiguiller les membres de l'équipe manquant de confiance en eux.

\section{Le livre comme projet éducatif}

La formation en équipe peut également permettre de proposer un projet partagé par toutes les professionnelles et durable dans le temps, favorisant ainsi une culture commune construite collectivement autour de la littérature jeunesse. Les professionnelles s'appuient beaucoup sur l'équipe et la formatrice indique comme nécessaire d'échanger autour du projet livre. Les échanges, l'interconnexion et la vision commune apparaissent ici indispensables à une formation efficace et des pratiques durables.

En outre, selon la littérature en sociologie du travail et des organisations, les connexions et échanges entre les membres de l'équipe semblent favoriser une amélioration des pratiques et du transfert des connaissances (Adler et Kwon, 2002). En effet, selon Berger et Luckmann (1966/1986), la construction d'un groupe social relève d'« une perspective qui part des individus et de leurs interactions, à l'intérieur d'un système donné ». Ainsi, l'individu construit son «identité » et ses apprentissages par son interaction avec la sphère sociale qui l'entoure. La dimension sociale et culturelle est introduite par ces auteurs "comme une matrice dans laquelle se construit l'organisation psychologique » (Berger et Luckmann, 1966/1986, p. 15). Une formation en équipe par la pratique réflexive pourrait donc conduire à la stabilisation d'un geste professionnel de manière durable et efficace, qui serait celui de l'usage du livre en accueil petite enfance.

Par le cheminement de cet article, nous avons ainsi compris que le suivi des seules préconisations de formateurs ou de théoriciens ne suffit pas à adopter une pratique efficace de la littérature jeunesse. C'est le partage en équipe, la réflexivité, la pratique et les ajustements quotidiens, en fonction des problématiques locales, en créant des situations favorables à l'émergence d'une culture commune stabilisée et à l'adhésion des enfants et des familles, qui vont permettre à la littérature jeunesse de vivre et de prendre place auprès du jeune enfant en structure d'accueil. Une recherche ultérieure sur la mise en place d'une formation en équipe auprès d'un échantillon plus important verrait ici son utilité afin de conforter nos conclusions.

\section{BIBLIOGRAPHIE}

Adler, P. S., et Kwon, S. W. (2002). Social capital: Prospects for a new concept. Academy of management review, 27(1), 17-40. 
Agence de Service et de Paiement (ASP). (2020). DEAP - Référentiel d'activité. Récupéré le 21 avril 2021 de https://vae.asp-public.fr/vaeinfo/deap-referentiel-dactivites

ARAGON, A. (2013). Des professionnels de la petite enfance et du livre cheminent ensemble. Spirale, 67(3), 70-77. doi :10.3917/spi.067.0070

Auger, N. (2011). Élèves nouvellement arrivés en France, réalités et perspectives pratiques en classe. Éditions des archives contemporaines.

Bagozzi, R. P. et Lee, K. H. (2002). Multiple Routes for Social Influence: The Role of Compliance, Internalization, and Social Identity. Social Psychology Quarterly, 65(3), 226-247.

Bardin, L. (2013). Chapitre III. La catégorisation. Dans L. Bardin, L'analyse de contenu (p. 150-168). Presses Universitaires de France.

Becker, E. A., et Gibson, C. C. (1998). Fishbein and Ajzen's theory of reasoned action: Accurate prediction of behavioral intentions for enrolling in distance education courses. Adult Education Quarterly, 49(1), 43-55.

Berger, P. et Luckmann T. (1986). The social construction of reality. A treatise in the sociology of knowledge [1966], trad. fr. La construction sociale de la réalité. Armand Colin.

BERGERON, E. (2009). Une rencontre mystérieuse : les bébés et les livres. Dans P. Ben Soussan (Dir), Les tout-petits et les livres (p. 77-84). Erès.

Best, F. (1973). Pour une pédagogie de l'éveil. Armand Colin.

Bigras, N. et Lemay, L. (2012). Petite enfance, services de garde éducatif et développement des enfants : État des connaissances. Presses de l'Université du Québec.

Bonnafé, M. (2001). Les Livres, c'est bon pour les bébés. Calmann-Lévy.

BONNAFÉ, M. (2019). Les bébés et les livres : questions au présent. In C. Haussin (Dir), Petite enfance : art et culture pour inclure (p. 41-54). ERES.

BOSSE-PLATIÈRE, S., DETHIER, A., FLEURY, C. et LOUTRE DU PASQUIER, N. (2011). 7. La

formation. Accueillir le jeune enfant : Un cadre de référence pour les professionnels (p. 245-290). ERES.

Bouissou, C., et Brau-Antony, S. (2005). Réflexivité et pratiques de formation. Carrefours de l'éducation, (2), 113-122.

Cabrejo-Parra, E. (2019). Premières transmissions de la langue orale. L'émerveillement de l'enfant face à la beauté des albums et des premiers récits. Colloque A.C.C.E.S. Médiathèque Marguerite Duras.

Carré, P. (2015). De l'apprentissage à la formation. Pour une nouvelle psychopédagogie des adultes. Revue française de pédagogie, 190(1), 29-40.

Chavepeyer, I. (2020). Langages artistiques et formation continue : chemin faisant.... Spirale, 93(1), 159.

Claridge, T. (2018). Dimensions of Social Capital - structural, cognitive, and relational. Social Capital Research et Training. Dunedin.

Commissariat général à la stratégie et à la prospective. (2014). Rapports et Documents : Lutter contre les stéréotypes filles-garçons. Récupéré le 03 juin 2021 de https://www.strategie.gouv.fr/ sites/strategie.gouv.fr/files/archives/CGSP_Stereotypes_filles_garcons_web.pdf

Centre de Ressources en Éducation de l'Enfance. (2021). Ressources pédagogiques : 0-4 ans, éveil au livre. Récupéré le 25 mai 2021 de https://crede-vd.ch/ressources-pedagogiques/0-4-ans/eveil-aulivre/ 
De Coster, M. (1990). Introduction à la sociologie. ( $\left.2^{\mathrm{e}} \mathrm{ed}\right)$. De Boeck-Wesmael.

DEPOVER, C. et MARCHAND, L. (2002). Chapitre 1 L'apprentissage tout au long de la vie et l'apprenant adulte. Dans C. DEPOVER et L. MARCHAND (Dir), E-learning et formation des adultes en contexte professionnel (p. 15-28). De Boeck Supérieur.

DETHIER, A., BOSSE-PLATIÈRE, S., FLEURY, C., et LOUTRE DU PASQUIER, N. (2011). Compétences et pratiques pour un accueil de qualité. Accueillir le jeune enfant : Un cadre de référence pour les professionnels (p. 139-197). ERES.

Diethert, A. P., Weisweiler, S., Frey, D., et Kerschreiter, R. (2015). Training motivation of employees in academia: developing and testing a model based on the theory of reasoned action. Motivationsforschung im Weiterbildungskontext (p. 29-50). Springer VS.

DUBAR, C. (2015). La socialisation : Construction des identités sociales et professionnelles. Armand Colin. Enfance et Musique. (2020). Programme de formation « Le livre et le tout-petit ».

Faingold, N. (2006). Explicitation des pratiques, réflexivité, construction identitaire. Analyse de pratiques, 78 .

Filliettaz, L. et Billett, S. (Eds.). (2015). Francophone perspectives of learning through work: conceptions, traditions and practices. Springer.

Fishbein, M. et Ajzen, I. (2010). Predicting and changing behavior: The Reasoned Action Approach. Taylor et Francis.

Flowers, H., Girolametto, L., Weitzman, E., et Greenberg, J. (2007). Promoting early literacy skills: Effects of in-service education for early childhood educators. Canadian Journal of Speech-Language Pathology and Audiology, 31(1), 6-18.

Hopstock, L. A. (2008). Motivation and adult learning: a survey among hospital personnel attending a CPR course. Resuscitation, 76(3), 425-430.

Lafortune L. (2015). L'accompagnement et l'évaluation de la réflexivité en santé : des applications en éducation et en formation. Presses de l'Université du Québec.

Lussi Borer, V., Durand, M. et Yvon, F. (Eds.). (2015). Analyse du travail et formation dans les métiers de l'éducation. De Boeck Supérieur.

Marcel, J.-F. (2002). Les pratiques comme objet d'analyse. Revue française de pédagogie, 138, 135-170.

Marchand Montanaro, G. (2013). Chapitre 3. Formations initiales : savoirs, normes et valeurs. Travailler en crèche, un métier (p. 59-74). Presses de l'École des hautes études en santé publique.

Meuret-Campfort, E. (2014). Dire la pénibilité du travail en crèche : Une enquête auprès d'auxiliaires de puériculture syndicalistes. Sociétés contemporaines, 95(3), 81-108.

Mondada, L. (2006). La compétence comme dimension située et contingente, localement évaluée par les participants. Bulletin suisse de linguistique appliquée, 84, 83-119.

Nières-Chevrel, I. (2002). Faire une place à la littérature jeunesse. Revue d'histoire littéraire de la France, vol. 102, 97-114.

Norwich, B., et Duncan, J. (1990). Attitudes, subjective norm, perceived preventive factors, intentions and learning science: Testing a modified theory of reasoned action. British Journal of Educational Psychology, 60(3), 312-321. 
Patras Mériaux, C. et Desrumaux, M.-C. (2018). «Parler bambin » à Grenoble : point de vue de professionnelles (p. 43-72). In P. Ben Soussan et S. Rayna (Dir), Le programme « Parler bamin »: enjeux et controverses. Erès.

PISELLI, N. et BRENGOU, R. (2019). Il était une fois... des livres, des enfants, des assistantes maternelles. Dans C. Haussin éd., Petite enfance : art et culture pour inclure (p. 123-133). Erès.

RAYNA, S. (2003). Regard sur les apprentissages des tout-petits. Enfances et Psy, n²4(4), 30-41.

Richer, S. et Vallerand, R. (1998). Construction et validation de l'Échelle du sentiment d'appartenance sociale (ÉSAS). Revue européenne de psychologie appliquée, vol. 48 n², 129-137. Presses de l'Université du Québec.

Schön, D. (1994). Le praticien réflexif. A la recherche du savoir caché dans l'agir professionnel. Traduit et adapté par J. Heynemand et D. Gagnon. Logiques.

Noblecourt, O. (2014). La lutte contre les inégalités commence dans les crèches. Récupéré le 20 avril 2021 de https://tnova.fr/...

Thollon-Behar, M. et Ignacchiti, S. (2019). De l'objet à l'histoire, le livre dans le développement de l'enfant. Enfances et Psy, 82(2), 39-48. https://doi.org/10.3917/ep.082.0039

Vertalier, M. (2006). Approche linguistique des textes de livres pour enfants : pour quels objectifs et quelles pratiques au cours de l'acquisition du langage? Dans : «Acquisition : implications didactiques ». Mélanges CRAPEL $n^{\circ} 29$.

Vinatier, I., Filliettaz, L., et Laforest (Eds.). (2018). L'analyse des interactions dans le travail : outil de formation professionnelle et de recherche. Raison et Passions.

\section{NOTES}

1. Du fait de la féminisation de ce métier où les femmes représentent plus de $99 \%$ du groupe professionnel en EAJE (Commissariat général à la stratégie et à la prospective, 2014), le féminin est employé dans ce texte pour les désigner.

\section{RÉSUMÉS}

La question de la formation des professionnelles petite enfance à l'usage du livre est encore peu développée bien que l'intérêt du livre pour le tout-petit ne soit plus à démontrer. En effet, les référentiels des formations professionnelles petite enfance ne mentionnent pas la littérature jeunesse. Cet article s'intéresse donc à la formation continue des professionnelles petite enfance à la littérature jeunesse dans un contexte de lutte contre les inégalités sociales. Dans une démarche inductive, nous cherchons à comprendre quelle pourrait être l'approche à privilégier pour former les professionnelles à cet effet. Notre enquête exploratoire auprès de professionnelles petite enfance met en évidence l'évocation de la dynamique de groupe pour la formation des professionnelles petite enfance à l'usage du livre jeunesse. Nous tentons ici de comprendre en quoi la formation professionnelle peut passer par une socialisation permettant une approche réflexive des pratiques et une professionnalisation des éducatrices à l'usage du 
livre auprès des jeunes enfants. Nous mobilisons un cadre théorique pluriel en étudiant l'équipe comme groupe social et l'Approche de l'Action Raisonnée ce qui nous permettra d'interroger des professionnelles de terrain en zone sensible. Cette analyse nous permettra de dégager des suggestions d'orientation pour former efficacement les professionnelles petite enfance à l'usage du livre.

The question of training early childhood professionals in the use of books is still underdeveloped, although the interest of books for toddlers is no longer to be demonstrated. Indeed, the standards for early childhood vocational training do not mention children's literature. This article therefore focuses on the continuing training of early childhood professionals in children's literature in a context of combating social inequalities. Using an inductive process, we try to understand what approach could be preferred to train professionals for this purpose. Our exploratory study among early childhood professionals highlights the importance of group dynamics in the training of early childhood professionals in the use of children's books. We attempt to understand how professional training can be achieved through a form of socialization that allows for a reflective approach of practices and the professionalization of book use with young children. We mobilize a plural theoretical framework by studying the team of professionals as a social group and relying on the method of the Reasoned Action Approach, which will enable us to interview professionals in sensitive areas. This analysis will enable us to draw up suggestions on how to effectively train early childhood professionals in the use of books.

\section{INDEX}

Mots-clés : formation, livre, équipe, petite enfance, social.

Keywords : Training, Book, Team, Early childhood, Social.

\section{AUTEURS}

\section{CATHERINE PERESSE SCHROEDER}

Université de Rouen, Université Lyon 2

\section{RAWAD CHAKER}

Université Lyon 2 